Aim of the study: To review management and outcomes in patients with stage I seminoma after orchidectomy.

Material and methods: Between 1979 and 2004 a total 292 patients with stage I seminoma were treated with adjuvant chemotherapy or radiotherapy or were placed on surveillance. Median age at diagnosis was 36 years (range 20-69), with median follow-up 76.5 months (range 11-294). Of the patients, 200 $(68.5 \%)$ were treated with adjuvant chemotherapy, 72 (24.6\%) were irradiated and $20(6.8 \%)$ were placed on surveillance.

Results: The probability of 5-year overall survival and relapse-free survival for the entire group was $100 \%$ and $95.1 \%$ respectively. The 5 -year relapse-free survival for adjuvant chemotherapy was $97.2 \%$, for radiotherapy $94.6 \%$, and $31.4 \%$ for the surveillance group. Of 24 (8.4\%) patients who had relapse in lymph nodes and/or internal organs, 14/20 patients were in the surveillance group. All patients who had a relapse were salvaged successfully with chemotherapy. The toxicity of chemotherapy and radiotherapy was acceptable. No severe reactions were observed.

Conclusion: Our results confirm the excellent prognosis for patients with stage I seminoma after orchidectomy treated with adjuvant chemotherapy or radiotherapy. The high rate of relapse in our surveillance group suggests the necessity of adjuvant treatment.

Key words: seminoma stage I, radiotherapy, chemotherapy, surveillance.

\section{Treatment of stage I seminoma: 25 years of experience}

\author{
Małgorzata Pilichowska ${ }^{1}$, Piotr Pęczkowski ${ }^{1}$, Dorota Rosińska-Okrasa ${ }^{2}$, \\ Barbara Trzaska², Anna Skowrońska-Gardas², Tomasz Demkow ${ }^{1}$
}

1Urooncology Department, Maria Skłodowska-Curie, Memorial Cancer Hospital, Warsaw, Poland

2Department of Radiotherapy, Maria Skłodowska-Curie, Memorial Cancer Hospital, Warsaw, Poland

\section{Introduction}

Germ-cell testicular tumours account for $1 \%$ of malignant neoplasms in men, with $60 \%$ of these tumours being seminomas [1]. In stage I testicular seminoma after orchiectomy the following options may be applied: regional lymph node radiotherapy, short-term chemotherapy based on platinum compounds or active surveillance [2-4].

Adjuvant radiotherapy was the standard practice in patients with stage I testicular seminoma within the last 50 years. Radiation was applied to the paraaortic and pelvic lymph nodes, where there is the greatest risk of micro metastases $[2,5,6]$. Mild early post-radiation reactions occur in approximately $60 \%$ of radiotherapy patients. About $5 \%$ of patients report late, chronic problems [6]. When selecting treatment options in patients with stage I testicular seminoma, the risk of secondary tumours in the area subjected to radiotherapy cannot be ignored either.

Following long-term follow-up of these patients, many authors have observed the increased risk of secondary tumours localised in the gastrointestinal tract, urinary bladder and the other testis $[7,8]$. The risk of leukaemia resulting from pelvic radiotherapy is also increased $[5,6,9]$. Therefore clinicians aim at reduction of the irradiation area and of the administered dose [4, 9]. The occurrence of adverse reactions following radiotherapy gave birth to the concept of the active surveillance of patients after orchiectomy for stage I testicular seminoma. Disease progression rate in these patients varies between 15 and $20 \%[10,11]$.

Then, if there are strictly defined prognostic factors for the early stage of testicular seminoma, approximately $80 \%$ of patients could avoid therapy and related complications [2, 5, 12]. Adjuvant carboplatin-based chemotherapy is the alternative for radiotherapy or strict surveillance in patients with stage I testicular seminoma. Chemotherapy reduces the risk of disease recurrence to approx. $10 \%[4,13,14]$.

\section{Material and methods}

From January 1979 to December 2004 the Institute of Oncology at Wawelska Street (Warsaw, Poland) and the Centre/Institute of Oncology at Ursynów (Warsaw, Poland) were attended by 328 patients with clinical stage I testicular seminoma. 36 patients were lost to follow-up. The group under analysis consists of 292 patients. Patients' age varied from 20 to 69 years (median 36 years). The follow-up period lasted from 11 to 294 months (median 76.5 months) from the day of diagnosis. All of the patients underwent surgical resection of a neoplastically changed testis. Histopathological test demonstrated typical seminoma in 279 patients, and anaplastic seminoma in 13 patients. All of the patients following surgery underwent imaging scans to exclude the presence of enlarged lymph nodes or metastases (chest X-ray, abdomen and 
pelvis ultrasound scan or chest, abdomen and pelvis computed tomography scans). Only 10 patients were not tested for $\alpha$-fetoprotein (AFP) and human chorionic gonadotropin (HCG) prior to orchiectomy (Table 1 ).

Selection of the post-orchiectomy treatment option depended primarily on the patient's decision, contraindications for the specific treatment option and therapeutic preferences changing over 25 years.

Following the resection of the testis with neoplastic changes, 20 patients (6.8\%) were subjected to active surveillance. 200 (68.5\%) patients were qualified for cytostatic therapy. The remaining 72 (24.6\%) underwent regional lymph node radiotherapy.

Chemotherapy was based on platinum compounds. 176 patients were administered one or two carboplatin courses. 13 patients were treated with the BEP (Cisplatin, Etoposide, Bleomycin) regimen, and 11 with the PVB (Cisplatin, Vinblastine, Bleomycin) regimen. One or two treatment courses were administered. BEP and PVB regimens were administered primarily in the 1980s. Since the 1990s carboplatin monotherapy has been applied (Table 2).

72 patients with stage I testicular seminoma were subjected to radiotherapy. 49 patients underwent para-aortic lymph node radiotherapy, and 23 para-aortic and pelvic lymph node radiotherapy. The total dose ranged from 24 to 36 Gy (Table 3). In the years 1979-1992 the area of para-aortic and pelvic lymph nodes on the tumour side was exposed to a radiotherapy dose above 24 Gy. Within the next years the area exposed to radiotherapy was reduced to para-aortic lymph doses, using the dose of 24-26 Gy.

Follow-up tests in the adjuvant therapy group under surveillance were conducted every 3 months within the first two years, every 6 months up to 5 years, and then once a year. Abdominal cavity and pelvis ultrasound and CT scans were ordered every 6-12 months, AFP and HCG tumour markers at each follow-up visit.

Table 1. Patient characteristics

\begin{tabular}{lc} 
No. of patients & 292 \\
\hline Age (median) & $20-69(36)$ \\
Histopathology & \\
$\quad$ seminoma classic & 279 \\
seminoma anaplastic & 13 \\
\hline Testis & \\
right & 144 \\
left & 148 \\
AFP & \\
$\quad$ normal & 280 \\
elevated & 2 \\
not tested & 10 \\
HCG & \\
normal & 260 \\
elevated & 22 \\
not tested & 10 \\
Treatment & \\
surveillance \\
chemotherapy \\
radiotherapy
\end{tabular}

Disease-free survival in the patients was evaluated using the Kaplan-Meier method.

\section{Results}

The follow-up period in patients following therapy was 28 to 294 months (median 80 months).

288 patients are alive, including 2 with disease recurrence. Two patients died from causes other than cancer, and 2 died from other tumours. Predicted 5-year survival for all of the patients was 100\%, disease-free survival 95.1\% (Fig. 1). Depending on the treatment option, predicted 5-year diseasefree survival was evaluated as $31.4 \%$ in the surveillance group, $97.2 \%$ in the chemotherapy group and $94.6 \%$ in the radiotherapy group (Fig. 2). The relation between disease-free survival and the administered dose was also explored, and was $100 \%$ for a 24 Gy dose and 93\% for a dose > 24 Gy.

Treatment failure manifested by lymph node or internal organ metastases was observed in 24 (8.2\%) patients. Time to disease progression ranged from 6 to 92 months. Disease recurrence was observed in 14/20 (70\%) patients under surveillance after orchiectomy. The principal reason for treatment failure in this group was para-aortic lymph node metastases, observed in 9 patients. In other cases disease recurrence occurred in 3 patients in pelvic lymph nodes, in 1 in the mediastinum and in 1 patient in inguinal lymph nodes. Only in $3(4.1 \%)$ radiotherapy patients was disease recurrence observed. One person had lung and mediastinal lymph node metastases, 1 had mediastinal lymph node metastases and 1 spinal cord and spine bone metastases. Treatment failure

Table 2. Chemotherapy characteristics

\begin{tabular}{|lc|}
\hline Chemotherapy regimens & No. of patients \\
\hline carboplatin & 176 \\
BEP & 13 \\
PVB & 11 \\
No. of cycles & \\
1 & 99 \\
2 & 99 \\
4 & 2 \\
\hline
\end{tabular}

Table 3. Radiotherapy characteristics

\begin{tabular}{|lc|}
\hline Target volumes & No. of patients \\
\hline para-aortic & 49 \\
para-aortic, iliac, inguinal & 23 \\
\hline Total dose & \\
24 Gy & 41 \\
25-30 Gy & 16 \\
31-36 Gy & 15 \\
\hline Dose/fraction & \\
1.5 Gy & 4 \\
1.8 Gy & 14 \\
2.0 Gy & 53 \\
2.2 Gy & 1 \\
Radiation energy & \\
4 MV & \\
9 MV & 15 \\
15 MV & 20 \\
y Co-60 & 22 \\
\hline
\end{tabular}




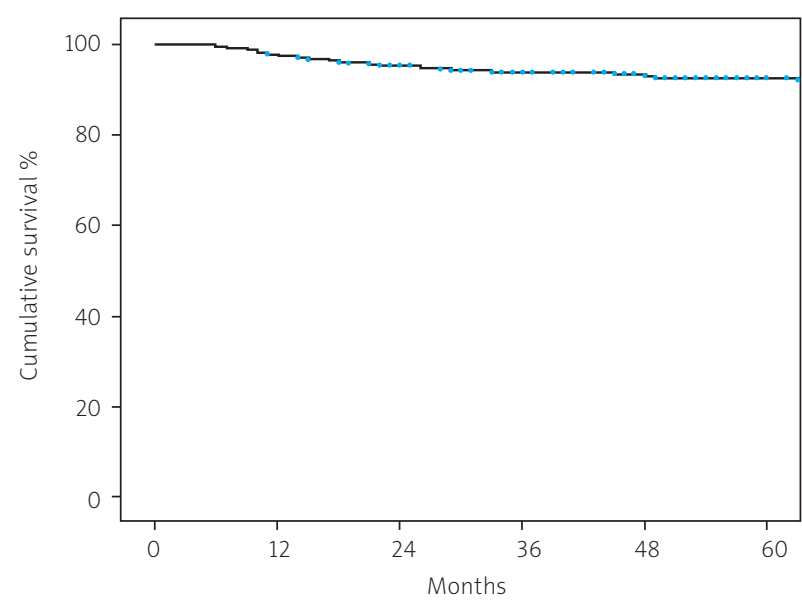

Fig. 1. Probability of 5-year disease-free survival for all patients

was also observed in 7 (3.5\%) patients subjected to chemotherapy, including para-aortic lymph node metastases in 2 patients, lung and mediastinal lymph node metastases in 3 patients and skeletal and lung metastases in 2 patients. None of the patients undergoing adjuvant radiotherapy had disease recurrence in the area exposed to radiotherapy. All of the patients with disease progression were subjected to effective chemotherapy. Chemotherapy toxicity was low. The most common symptoms included nausea and/or vomiting, observed in 8 patients. Two patients complained of abdominal pain, in 2 changes in the oral mucosa were observed, in 8 moderate thrombocytopenia, and in 2 leukopenia was observed. Side effects manifesting as mild nausea and/or vomiting, abdominal pain, diarrhoea, weakness and leukopenia occurred in $42 \%$ of the patients subjected to radiotherapy. The severity of early post-radiation reactions was degree 1 according to the RTOG/EORTC scale. In none of the patients subjected to radiotherapy were late post-radiation symptoms noted during surveillance. Under long-term surveillance in 15 patients treated for stage I testicular seminoma other cancers were reported, including 12 patients subjected previously to cytostatic therapy, and 3 subjected to radiotherapy. No other cancers were observed in patients under strict surveillance. In the chemotherapy group 6 testicular tumours were observed, among which 4 patients were diagnosed with seminoma, and 2 with embryonic cancer. Lung cancer was diagnosed in 2 patients, thyroid cancer in 2 patients, renal cancer in 1 patient and small intestine cancer in 1 patient. In the radiotherapy group no tumours of the other testis were observed. In this group lung, thyroid and small intestine cancer was observed.

\section{Discussion}

The prognosis for patients with stage I testicular seminoma is very good.

In our material survival was $100 \%$ and disease-free survival $95.1 \%$. Similar results were obtained by other authors $[2,6,10,15]$.

Only in 3/72 radiotherapy patients was post-orchiectomy disease recurrence observed. None of them was diagnosed with disease recurrence in the area exposed to radiotherapy. Although $68 \%$ of patients were subjected to radiother-

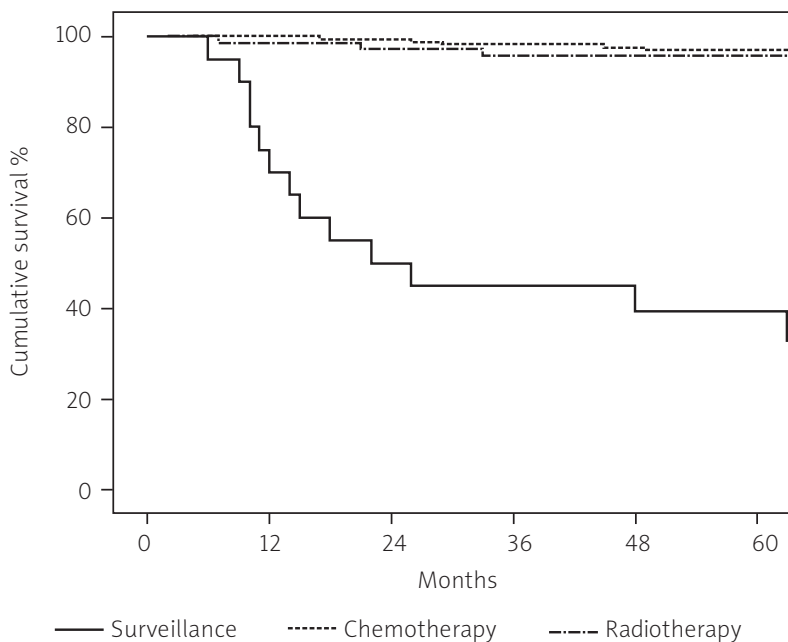

Fig. 2. Probability of 5-year disease-free survival depending on the treatment option

apy of the para-aortic lymph node area only, disease progression was not related to the pelvic lymph nodes. Results of the controlled clinical trial TE10 (478 patients) published by Fossa et al. [6], comparing the effects of radiotherapy in patients subjected to para-aortic lymph node radiotherapy and both to para-aortic and pelvic lymph node radiotherapy, demonstrated an identical rate of 5-year disease-free survival (96\%). Disease recurrence in the pelvis was observed in 4 patients subjected to para-aortic lymph node radiotherapy. In another controlled clinical trial, TE18 (625 patients), Jones et al. [16] presented the results of the therapy in patients subjected to para-aortic lymph node radiotherapy at a dose of $20 \mathrm{~Gy}$ and $30 \mathrm{~Gy}$. Also in this case disease-free survival was comparable in both study groups and amounted to $96.8 \%$ and $95.1 \%$, respectively. In our material the difference between the dose 24 Gy and > 24 Gy was greater and it was estimated at $100 \%$ and $93 \%$. Many authors suggest that the reduction of the radiation field and the reduction of the administered dose diminish the risk of secondary tumours in the radiation field and in the other testis $[4,6,9,15]$.

Van den Belt-Dusebout et al. [8] suggest that both radiotherapy and cisplatin-based chemotherapy increase the risk of secondary tumours and cardiovascular diseases (1.8-fold and 1.9-fold for radiotherapy and chemotherapy, respectively). Ruther et al. [7] estimated the relative risk of secondary tumours in radiotherapy patients at 4.8, and for the occurrence of seminoma in the other testis at 44.8. A smaller radiation field results in lower early and late toxicity. In our centre $42 \%$ of radiotherapy patients manifested early side effects, mostly mild nausea and/or vomiting. No late postradiation symptoms were observed. Similar results were presented by other authors $[15,17]$. The alternative treatment option for patients with stage I testicular seminoma is strict surveillance, allowing avoidance of toxicity symptoms and potential secondary tumours in young patients subjected to chemotherapy or radiotherapy. Probability of disease recurrence in the patients under surveillance is estimated at $15-20 \%$, mostly in the group with adverse risk factors such as tumour size $>4 \mathrm{~cm}$ and tumour extension in the rete testis 
$[6,10,12]$. Strict follow-up requires long-term monitoring of the patients, high patient compliance and sufficient financial resources in healthcare facilities for regular diagnostic tests $[6,10,12,18]$. It is also worth noting patients' exposure to radiation generated by frequent computed tomography scans, and consequently the possibility to induce secondary tumours, as was observed by Tarin et al. [19]. It is estimated that $60-70 \%$ of all failures during active surveillance are localised in para-aortic lymph nodes $[2,4,5,10]$. In the active surveillance group, which we analysed, the rate of failures was high and amounted to $70 \%$. It is probably due to the incomplete diagnostics in patients at the turn of the 1980s and 90s. The largest group in our centre consisted of patients with stage I testicular seminoma subjected to adjuvant chemotherapy after orchiectomy. Failures in this group of patients amounted to $4 \%$. Comparable results were reported by other centres $[4,5,14]$. The observed toxicity of cytostatic therapy was low. It related to $10 \%$ patients only and was manifested mostly by mild nausea and/or vomiting, as well as thrombocytopenia and leukopenia. The majority of authors emphasise low toxicity of chemotherapy and state that 2 carboplatinum courses offer more effective protection against disease progression $[13,14]$. Selection of the treatment option in patients with stage I testicular seminoma after orchiectomy is still under discussion. Data from the multicentre controlled clinical trial TE 19, comparing the efficacy of adjuvant radiotherapy and chemotherapy, demonstrated comparable 5-year disease-free survival, amounting to 96\% and $95 \%$, respectively [14].

In conclusion: our findings support very good prognosis for patients with stage I testicular seminoma after orchiectomy. The use of radiation fields limited to para-aortic lymph nodes and the total dose of 24 Gy reduces early and late toxicity and poses lower risk of secondary tumours. Chemotherapy using carboplatinum offers a good alternative for radiotherapy. As our analysis shows, active surveillance may involve a great risk of disease recurrence, and therefore in these patients it seems reasonable to use adjuvant therapy.

\section{References}

1. Zatoński WA. Nowotwory złośliwe w Polsce. Centrum Onkologii - In stytut im. M. Skłodowskiej-Curie w Warszawie, Warszawa 1995

2. Horwich A, Dearnaley DP. Treatment of seminoma. Semin Oncol 1992; 19: 171-80.

3. Madej G. Chemioterapia dorostych i dzieci. PZWL, Warszawa 1992

4. Martin J, Chung P, Warde P. Treatment options, prognostic factors and selection of treatment in stage I seminoma. Onkologie 2006; 29: 592-598.

5. Albers P. Management of stage I testis cancer. Eur Urol 2007; 51: 34-43.

6. Fossa SD, Horwich A, Russell JM, et al. Optimal planning target volume for stage I testicular seminoma: A Medical Research Council randomized trial. Medical Research Council Testicular Tumor Working Group. J Clin Oncol 1999; 17: 1146.

7. Ruther U, Dieckmann KP, Bussar-Maatz R, Eisenberger F. Second ma lignancies following pure seminoma. Oncology 2000; 58: 75-82.

8. van den Belt-Dusebout AW, de Wit R, Gietema JA, et al. Treatmentspecific risks of second malignancies and cardiovascular disease in 5-year survivors of testicular cancer. J Clin Oncol 2007; 25: 4370-8.

9. Zwahlen DR, Martin JM, Millar JL, Schneider U. Effect of radiotherapy volume and dose on secondary cancer risk in stage I testicular seminoma. Int J Radiat Oncol Biol Phys 2008; 70: 853-8.
10. Alomary I, Samant R, Gallant V. Treatment of stage I seminoma: a 15-year review. Urol Oncol 2006; 24: 180-3.

11. Mead GM, Fossa SD, Oliver RT, et al. Randomized trials in 2466 patients with stage I seminoma: patterns of relapse and follow-up. J Natl Cancer Inst 2011; 103: 241-9.

12. Warde P, Specht L, Horwich A, Oliver T, Panzarella T, Gospodarowicz M, von der Maase $\mathrm{H}$. Prognostic factors for relapse in stage I seminoma managed by surveillance: a pooled analysis. J Clin Oncol 2002; 20: 4448-52.

13. Dieckmann KP, Bruggeboes B, Pichlmeier U, Kuster J, Mullerleile U, Bartels $\mathrm{H}$. Adjuvant treatment of clinical stage I seminoma: is a single course of carboplatin sufficient? Urology 2000; 55: 102-6.

14. Oliver RT, Mason MD, Mead GM et al.; MRC TE19 collaborators and the EORTC 30982 collaborators. Radiotherapy versus single-dose carboplatin in adjuvant treatment of stage I seminoma: a randomised trial. Lancet 2005; 366: 293-300.

15. Niazi TM, Souhami L, Sultanem K, Duclos M, Shenouda G, Freeman C. Long-term results of para-aortic irradiation for patients with stage I seminoma of the testis. Int J Radiat Oncol Biol Phys 2005; 61: 741-4.

16. Jones WG, Fossa SD, Mead GM, Roberts JT, Sokal M, Horwich A, Stenning SP. Randomized trial of 30 versus 20 Gy in the adjuvant treatment of stage I Testicular Seminoma: a report on Medical Research Council Trial TE18, European Organisation for the Research and Treatment of Cancer Trial 30942 (ISRCTN18525328). J Clin Oncol 2005; 23: 1200-8

17. Niewald M, Freyd J, Fleckenstein J, Wullich B, Rube C. Low-dose radiotherapy for Stage I seminoma - long-term results. Int J Radiat Oncol Biol Phys 2006; 66: 1112-9.

18. Chang SS, Roth B. Treatment of clinical stage I germ cell tumors. Urology 2002; 59: 173-9.

19. Tarin TV, Sonn G, Shinghal R. Estimating the risk of cancer associated with imaging related radiation during surveillance for stage I testicular cancer using computerized tomography. J Urol 2009; 181: 627-32

\section{Address for correspondence}

\section{Małgorzata Pilichowska}

Urooncology Department, Maria Skłodowska-Curie, Memorial Cancer Hospital,

Roentgena 5

02-781 Warszawa, Poland

e-mail: radioterapia@o2.pl

Submitted: $\quad$ 18.03.2011

Accepted: $\quad$ 2.02.2012 